\title{
Spinal cord infarction: clinical and magnetic resonance imaging findings and short term outcome
}

\section{Masson, J P Pruvo, J F Meder, C Cordonnier, E Touzé, V de la Sayette, M Giroud, J L Mas, D Leys, for the Study Group on Spinal Cord Infarction of the French Neurovascular Society}

J Neurol Neurosurg Psychiatry 2004;75:1431-1435. doi: 10.1136/jnnp.2003.031724

See end of article for authors' affiliations

Correspondence to:

C Masson, Department of

Neurology, Beaujon

Hospital, $100 \mathrm{bd}$ du

Général Leclerc, 92110

Clichy, France;

catherine.masson@

bjn.ap-hop-paris.fr

Received

3 November 2003

In revised form

13 January 2004

Accepted 15 January 2004

\begin{abstract}
Background: Most studies on spinal cord infarction have been conducted in single centres; they usually consisted of case reports, or of larger series of patients recruited over a large period of time, with heterogeneous diagnostic procedures. Therefore, the clinical and radiological presentation of spinal cord infarcts and their short term outcome remain poorly understood.

Objective: To define clinical and magnetic resonance imaging (MRI) findings, and short term outcome in patients with spinal cord infarcts.

Methods: The authors prospectively included patients within 10 days of onset. An MRI scan was required and repeated when initially normal.

Results: Twenty eight consecutive patients were included over a 24 month period in 16 neurological centres. The infarct was cervical in seven patients, thoracic in three, thoracolumbar in 15, and restricted to the conus in three. On axial MRI scans the infarct was located in the central territory of the anterior spinal artery in 21 patients, and in the peripheral arterial territory in three. At month two, 15 patients had a good outcome and 13 had a poor outcome, including three deaths. Patients who, at onset, could not walk, had bladder dysfunction, or proprioceptive deficits were more likely to have a poor outcome. At month two, pain had occurred in 10 of the 25 survivors and was associated neither with the initial severity, nor the extent of the infarct on MRI.

Conclusion: The two month outcome mainly depends on the initial severity of the neurological deficit; however, a few patients with a severe impairment at onset had a good outcome, especially when proprioception remained normal at onset. The study does not support the hypothesis that pain occurs more frequently in small spinal infarcts.
\end{abstract}

S pinal cord infarcts are rare, and the most recent publications were case reports describing clinical and radiological features, or unusual causes. ${ }^{1-8}$ The few series of cases were retrospective, or did not systematically perform a magnetic resonance imaging (MRI) scan at the acute

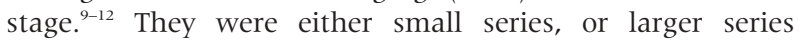
collected over a long period of time with heterogeneous diagnostic work up. ${ }^{9-12}$ For these reasons, the clinical and radiological presentation of spinal cord infarcts, and their short term outcome remain poorly known.

The aim of this prospective, multicentre study was to describe the clinical and radiological presentation of spinal cord infarcts and their short term outcome in 28 consecutive patients recruited over a two year period, who underwent a homogeneous diagnostic work up with MRI at the acute stage.

\section{PATIENTS AND METHODS}

Clinical neurologists from University and non-University French hospitals were asked to participate in this prospective study. Neurologists from 16 centres accepted and were active. The study was observational: no test or treatment was required that was not part of the current practice in the participating centres and the management of patients was left to the discretion of local investigators. The only two investigations that were mandatory were clinical and MRI investigations. The recruitment was conducted over a two year period from 15 January 2001 to 14 January 2003.

\section{Inclusion and exclusion criteria}

Patients were included within 10 days of the onset of symptoms. Inclusion criteria were: (1) the occurrence of a spinal cord syndrome of acute or subacute onset (that is, delay from onset to maximum intensity $<72$ hours) and (2) a spinal cord MRI scan either normal or showing on T2 weighted images a focal high intensity signal compatible with a spinal cord infarct. Exclusion criteria were: clinical, biological, or MRI findings suggesting an acute compressive myelopathy or an acute myelitis related to sclerosis, systemic disease, or parainfectious.

\section{Methods}

Patients were included by neurologists. Data were collected by means of a standardised questionnaire. Clinical data recorded were: sex; age; previous transient symptoms suggesting spinal transient ischaemic attacks; clinical symptoms at onset including back pain; ability to walk (with one aid or less, with two aids, or impossible); motor deficit; touch and pain perception; temperature discrimination; joint position sense; and bladder function. Quantitative sensory testing or somatosensory evoked potentials were not required. According to the initial neurological assessment, patients were categorised as initially "severely impaired" if they could not walk (or could only walk with two aids), and had bladder dysfunction needing catheterisation.

All patients had a MRI scan within 10 days of clinical onset, and when the first MRI scan was normal, a delayed MRI was performed. The MRI protocol required Tl and T2 weighted sagittal images visualising the entire length of the spinal cord, and T2 axial weighted images at the level of the

Abbreviations: CSF, cerebrospinal fluid; ECG, electrocardiogram; MRI, magnetic resonance imaging. 
lesion. All spinal cord MRI scans were reviewed by consensus by two experienced neuroradiologists (JPP and JFM).

All patients had routine blood tests, an ECG, and an evaluation of vascular risk factors: arterial hypertension (systolic blood pressure of $\geqslant 160 \mathrm{~mm} \mathrm{Hg}$ or diastolic blood pressure of $\geqslant 90 \mathrm{~mm} \mathrm{Hg}$, or current treatment with antihypertensive drugs); diabetes mellitus (fasting blood glucose of $>126 \mathrm{mg} / \mathrm{dl}$ or current use of hypoglycaemic agents); dyslipidaemia (fasting cholesterol serum level of $>250 \mathrm{mg} / \mathrm{dl}$, serum level of triglyceride of $>150 \mathrm{mg} / \mathrm{dl}$, or current use of lipid lowering medication); peripheral artery disease with intermittent claudication; atrial fibrillation (ECG); previous stroke (medical history and reports); or myocardial infarction (medical history and reports and ECG). Silent strokes were not taken into account because brain imaging was not required, but silent myocardial infarcts found on ECG were classified as myocardial infarcts. The initial assessment also included a search for the following events that may have occurred less than one week before onset: spinal manipulation; strenuous effort; aortic surgery; diagnostic or therapeutic angiography. The aorta (in thoracic and lumbar infarcts) and cervical arteries (in cervical infarcts) were evaluated by non-invasive imaging methods (any combination of ultrasounds, computed tomography or MRI scans). Transoesophageal echography and serological tests for coagulation, infectious and connective diseases were optional, and performed only when considered as necessary by the local investigators. Cerebrospinal fluid (CSF) examination was mandatory when no clearly definite cause of spinal cord infarct was present, and patients with inflammatory changes were excluded.

The short term outcome was assessed at month two (follow up 60 days), concerning mortality, walking ability, bladder function, and pain. The severity of pain was graduated from zero to 10 by a visual analogue scale, but its qualitative description was not evaluated.

A good outcome was defined as the ability to walk without help or with only one aid, and the absence of any bladder dysfunction that needed catheterisation. A poor outcome was defined as death, inability to walk or walking with two aids, or bladder dysfunction needing intermittent catheterisation or indwelling catheter.

\section{Statistics}

To identify variables that can influence the functional outcome, we compared demographic factors and initial clinical and MRI features between patients with good and poor outcomes. We used $\chi^{2}$ test, with Yates correction or Fisher's exact test when appropriate, to compare qualitative variables, and the non-parametric Mann-Whitney $U$ test to compare quantitative variables. Because of the small number of patients we did not perform any multivariate analysis. We performed statistics with the SPSS 9.0 for Windows program (SPSS Inc, Chicago, IL, USA).

\section{RESULTS}

\section{Study population}

During the study period, 28 patients were included (20 men; median age 62 years, range 20 to 85 years). Nineteen patients had at least one vascular risk factor (table 1). A definite cause was found in 11 patients (table 2). The infarct occurred after a strenuous effort in one patient. Four patients had no cause, nor any vascular risk factor.

\section{Clinical features}

Clinical features are detailed in table 3. No patient reported symptoms of previous spinal cord transient ischaemic episode. Disturbances of proprioception were never isolated
Table 1 Vascular risk factors in the study population. Nine patients were free of any risk factors, five patients had one risk factor, eight had two risk factors, and six had three or four risk factors. See text for definitions of risk factors. Values are number of patients

\begin{tabular}{lc}
\hline Risk factors & Patients $(\mathbf{n}=\mathbf{2 8})$ \\
\hline Arterial hypertension & 15 \\
Diabete mellitus & 5 \\
Dyslipidaemia & 8 \\
Atrial fibrillation & 2 \\
Peripheral-artery disease & 5 \\
Previous stroke & 0 \\
Previous myocardial infarct & 4 \\
\hline
\end{tabular}

and were always associated with some degree of loss of pain and temperature sensations.

\section{MRI findings and location of the infarct}

The first MRI scan was performed within 24 hours in nine patients, within five days in 23 patients, and within 10 days in all. The first MRI showed the infarct in 24 patients: in the four patients with an initial normal MRI (day one in one patient, at day two in three), the second MRI performed 10 to 21 days later remained normal, even after contrast enhancement. These four patients had a typical clinical syndrome of acute anterior spinal cord infarct. Two of them had a definite vascular cause and the two others had normal CSF examination and brain MRI. The location and extent of the infarcts could therefore be clearly defined in 24 patients on T2 weighted MRI images in the sagittal plane (fig l). The four patients with normal MRI scans had a thoracolumbar infarct, as defined on the basis of the clinical examination. The location of the increased signal intensity in the central or peripheral arterial territory of the spinal cord could be identified in the 24 patients in whom a lesion was seen on $\mathrm{T} 2$ weighted images in the axial plane (figs 2 and 3). Twenty one infarcts were located in the central territory of the anterior spinal artery: of these 21 patients, 14 had an extensive hyperintense signal involving the grey matter and the adjacent central white matter, and seven had a limited hyperintense signal more prominent in the anterior grey matter, leading to an "owl's eyes" pattern. The three remainders had a lesion located in the peripheral arterial territory of the spinal cord: in one, the lesion was bilateral, in the territory of the posterior spinal arteries, and in the other two, the lesion was located in the white matter of one lateral column, and spared the posterior column. Of the 21 patients

Table 2 Causes of spinal cord infarcts in the study population

\begin{tabular}{lc}
\hline Causes & $\begin{array}{c}\text { Patients } \\
(\mathbf{n = 2 8})\end{array}$ \\
\hline Aortic disease* $^{*}$ & \\
Aneurysm & 4 \\
Thrombosis & 1 \\
Dissection & 1 \\
Aortic surgery & 2 \\
Unilateral vertebral artery occlusion (atheroma)t & 1 \\
Thyro-bicervico scapular artery occlusiont & 1 \\
Thoracic wound (stab)* & 1 \\
No cause & 17 \\
\hline
\end{tabular}

*Of 21 patients with thoracic or thoracolumbar infarct. †Of seven patients with cervical infarcts.

$\ddagger$ Twelve of 21 patients with thoracic or thoracolumbar infarcts and five of seven patients with cervical infarcts. 


\begin{tabular}{|c|c|}
\hline Characteristics at onset & Patients ( $n=28$ ) \\
\hline \multicolumn{2}{|l|}{ Onset of symptoms* } \\
\hline$\leqslant 30$ minutes & 11 \\
\hline$\leqslant 12$ hours & 18 \\
\hline$\leqslant 72$ hours & 28 \\
\hline Back pain at onset & 23 \\
\hline Motor deficit & 28 \\
\hline Tetraparesis & 7 \\
\hline Paraparesis & 10 \\
\hline Paraplegia & 9 \\
\hline Unilateral deficit of limb & 2 \\
\hline \multicolumn{2}{|l|}{ Walking } \\
\hline Impossible & 18 \\
\hline Possible with 2 aids & 2 \\
\hline Possible with 1 aid & 7 \\
\hline Possible without aid & 1 \\
\hline Indwelling catheter & 21 \\
\hline Sensory impairment & 25 \\
\hline Spinothalamic $†$ & 25 \\
\hline Proprioceptiveł & 15 \\
\hline Severe impairment\$ & 19 \\
\hline \multicolumn{2}{|c|}{$\begin{array}{l}\text { *Cumulative rates from onset to maximum intensity. } \\
\text { †Loss of pain and temperature sensation. } \\
\text { flmpairment in the sense of position. } \\
\text { SInability to walk alone or with } 1 \text { aid, and bladder } \\
\text { dysfunction needing catheterisation. }\end{array}$} \\
\hline
\end{tabular}

with an infarct located in the central territory of the anterior spinal artery, 20 had a motor deficit, a sensory spinothalamic loss below the level of the infarct, and bladder dysfunction, of which 12 had also a proprioceptive impairment below the lesion. The only patient without sensory abnormality had an infarct restricted to the grey matter of the conus.

\section{Cerebrospinal fluid}

Cerebrospinal fluid was studied in 15 patients, and was found to be normal in seven and showed a slightly increased protein level up to $0.75 \mathrm{~g} / \mathrm{l}$ in eight, without pleocytosis, increased CSF IgG index, or oligoclonal bands. CSF was not examined in the 11 patients with a definite cause of spinal cord infarct and in two with the typical "owl's eye" MRI pattern and multiple vascular risk factors.

\section{Patients}

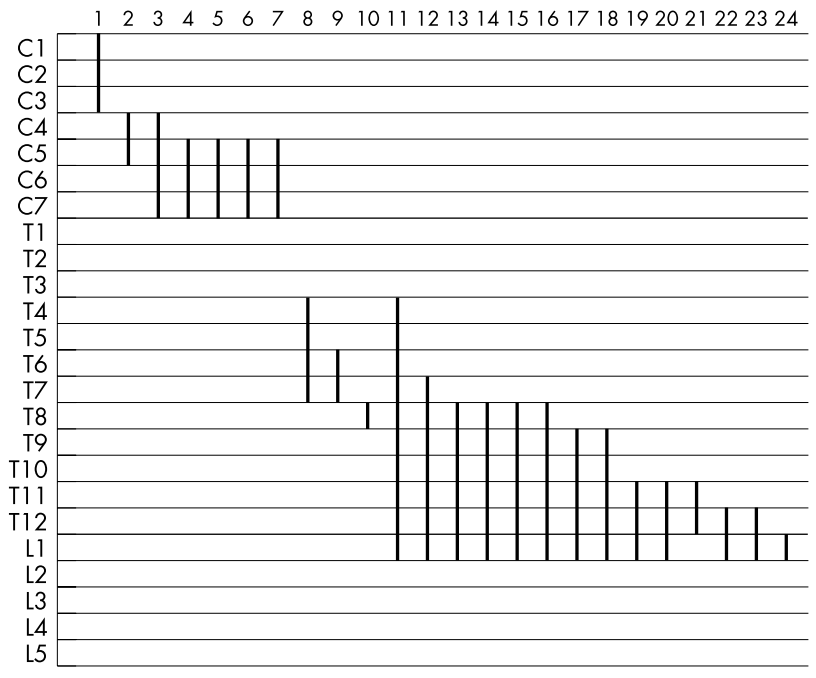

Figure 1 Location and extent of the infarcts, related to vertebral bodies, evaluated in 24 patients whose MRI showed the infarct. Black areas represent the spinal areas involved in the infarct in each patient on T2 weighted MR images.

\section{Two month outcome and associated factors}

Fifteen patients had a good outcome, and 13 a poor outcome including three deaths. The causes of death were infection in two patients, and a brainstem infarct in one. The outcome was not associated with the level or extent of the infarct on MRI. At month two, patients who were classified in the good outcome group were less likely to have been unable to walk or walk only with two aids $(p=0.007)$, or to have proprioceptive impairment $(\mathrm{p}=0.001)$ or bladder dysfunction $(p=0.016)$ at onset, but they did not differ from those who had a poor outcome for sex, age, other neurological deficits, level of the infarct, arterial territory involved, and presence of a definite cause. In the subgroup of 19 patients classified as initially "severely impaired", a good outcome was found in five of the six patients who had no proprioceptive impairment at onset, and in one of the 13 patients who had a proprioceptive impairment at onset $(p=0.003)$. Of the 25 survivors at month two, 10 had developed pain below the level of the lesion: pain was not associated with clinical and MRI features at onset or disability at month two. All patients who developed pain had sensory spinothalamic impairment below the lesion at onset, but only 10 of the 22 patients with initial spinothalamic disturbances developed pain $(\mathrm{p}=0.250)$.

\section{DISCUSSION}

Our study has shown that spinal cord infarcts: (1) had a clearly definite cause in less than $50 \%$ of patients; (2) were more frequently located in the central territory of the anterior spinal artery; (3) more often involved the dorsolumbar area, then the cervical area, and were rarely limited to the midthoracic spinal cord; (4) had a worse two month outcome when proprioceptive impairment, walking impairment, or bladder dysfunction were present at onset, and (5) frequently led to residual pain, irrespective of the extent of the infarct.

Although vascular risk factors were present in most patients, less than $50 \%$ of patients had a definite cause. However, aortic surgery was probably underrepresented in this series because vascular surgeons were not primarily involved in this study.

The location of infarcts defined on MRI is similar to that reported in old neuropathological studies, ${ }^{13}$ and in a recent MRI study. ${ }^{14}$ Although previous case reports, or reports of small series might suggest a high frequency of small infarcts located in the peripheral arterial territory, ${ }^{1-8}$ only three of our 28 patients had infarcts sparing the central territory of the anterior spinal artery. In most patients, the infarct was located in the central territory of the anterior spinal artery. In seven of these patients, MRI showed the "owl's eyes" pattern, suggesting that the infarct involved primarily the anterior part of the grey matter. ${ }^{15}$

In a recent retrospective study of 36 patients with spinal cord infarcts, the initial severity of the neurological deficits was the best predictor of outcome. ${ }^{12}$ We also found a relation between the functional outcome at month two and the severity of the neurological deficit at onset. However, a few patients who were unable to walk and required an indwelling catheter at admission finally had a good outcome. Similar findings were reported by Monteiro et al. ${ }^{9}$ Therefore, it would be useful to have other predictors of early functional recovery. Mawad et al ${ }^{15}$ suggested that an "owl's eyes" pattern on MRI could be associated with a better outcome, but our study does not support this finding. However, at an early stage, the extent of the infarct can hardly be defined, because hyperintense signal abnormalities on $\mathrm{T} 2$ weighted MRI scans may be due in part to mechanisms other than infarction-for example, vasogenic oedema. Moreover, the small number of patients in all subgroups led to a low statistical power. 

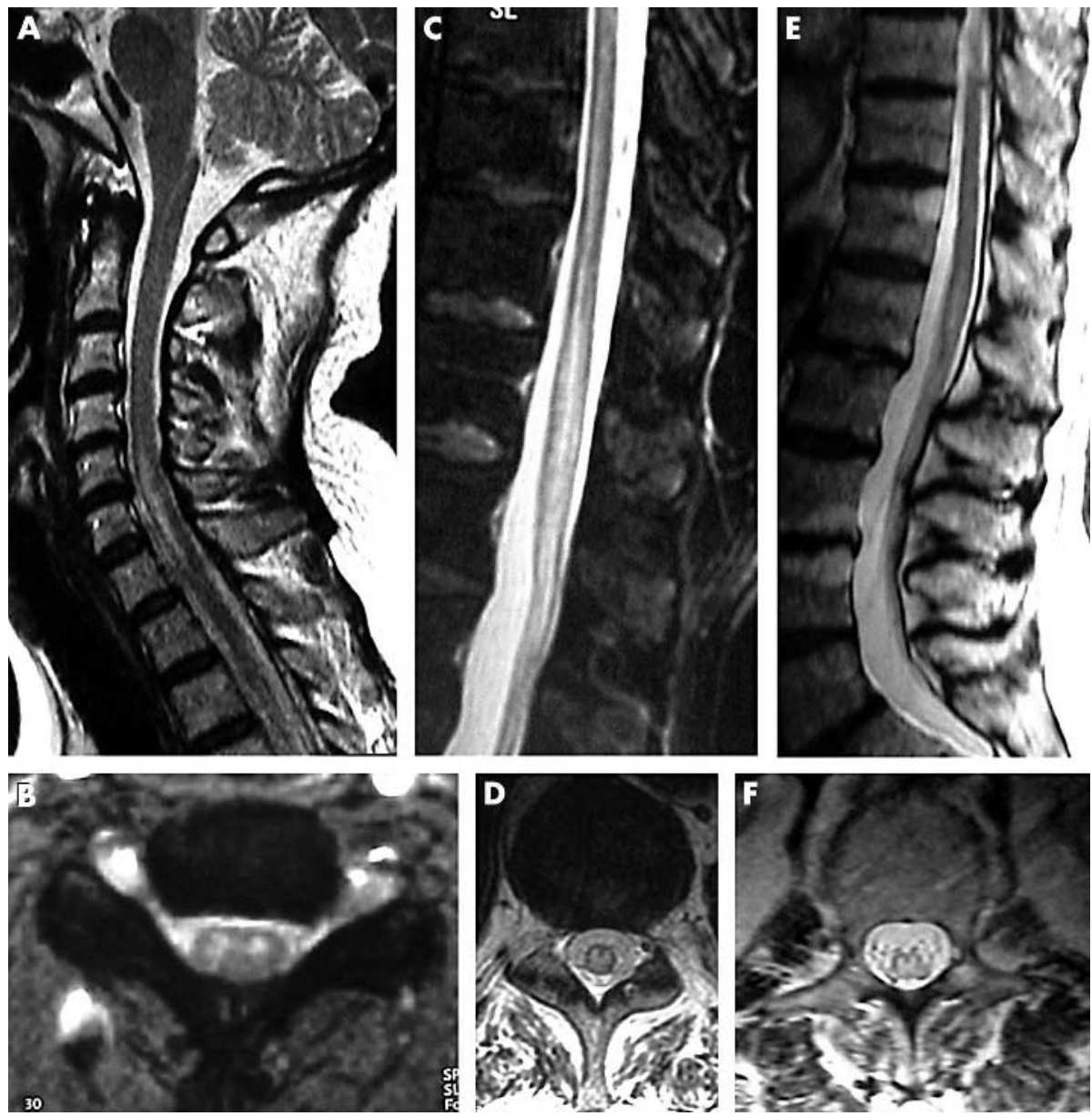

Figure 2 Spinal cord infarcts in the

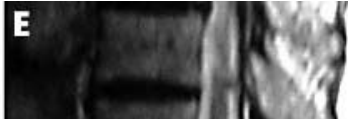
central territory of the anterior spinal artery. All scans were provided by $\mathrm{T} 2$ weighted sequence. ( $A$ and $B$ ) Cervical spinal cord infarct. (A) Sagittal: high intensity lesion between C5 and C7 spinal levels. (B) Axial: hyperintense signal predominated on anterior grey matter leading to the "owl's eyes" pattern. (C and D) Thoracolumbar spinal cord infarct. (C) Sagittal: high intense lesion between Th 8 and L1 spinal levels. (D) Axial: hyperintense signal with involvement of grey matter and adjacent central white matter. ( $E$ and $F$ ) infarct of the conus. (E) Sagittal: high intense lesion at L1 spinal level. (F) Axial: hyperintense signal in the centre of the conus.
The absence of proprioceptive impairment at onset was associated with a better outcome: all patients but three who had a good outcome had no proprioceptive impairment at
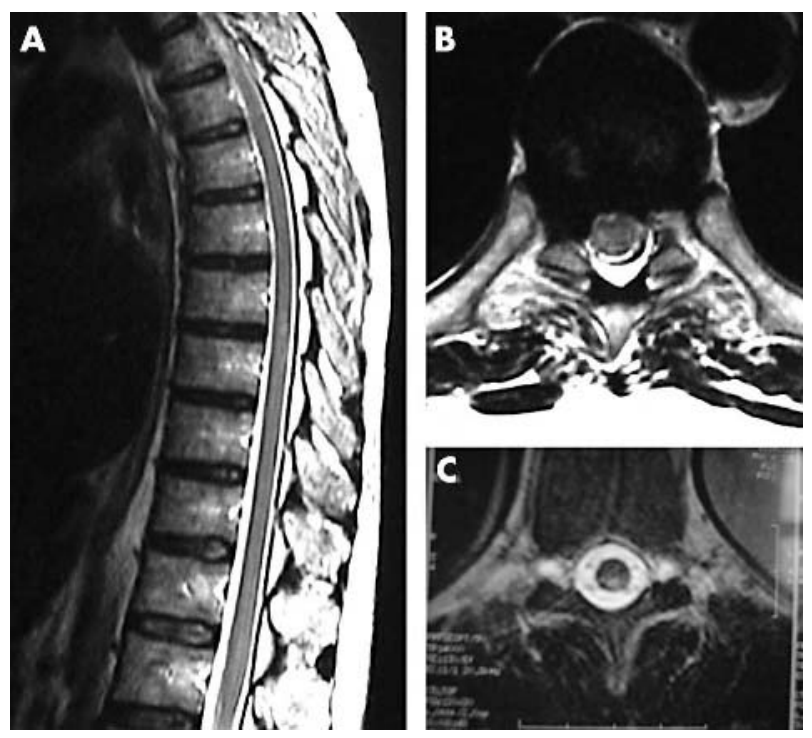

Figure 3 Spinal cord infarcts in the peripheral territory. All scans were provided by $\mathrm{T} 2$ weighted sequence. (A) Sagittal: small high intensity lesion at T8 spinal level. (B) Axial: hyperintense signal in the right posterolateral region of the cord, sparing the posterior column. (C) Axial: bilateral hyperintense signal, in the posterior region of the spinal cord. onset. Proprioception, conveyed by the dorsal columns, is usually spared in anterior spinal artery syndromes, but not in all patients ${ }^{15}: 12$ of the 21 patients with an anterior spinal artery territory infarct on MRI had some degree of proprioceptive impairment at onset. In such cases, proprioceptive impairment is usually explained by involvement of the most inner part of the dorsal columns that belongs to the central territory of the anterior spinal artery. However, dorsal columns have no exclusivity in proprioceptive conduction. ${ }^{16}$ Only fine discriminative functions such as graphaestesia are carried on exclusively by the dorsal columns. ${ }^{16}$ Loss of proprioception requires an additional lesion in the most posterolateral part of the lateral column that includes the dorsal spinocerebellar tract. ${ }^{16}{ }^{17}$ When proprioceptive impairment is present in anterior spinal artery syndromes, this may suggest that the infarct is more extensive and involves the inner part of the dorsal columns and the posterolateral part of the lateral columns. ${ }^{16}$ The worst outcome observed in our study in patients who had proprioceptive impairment at onset supports this hypothesis.

Pain is a frequent disabling feature two months after a spinal cord infarct ${ }^{10}{ }^{11}$ : nearly half of our patients developed pain irrespective of the severity at onset. Seven of the 10 patients of Pelser and van Gijn ${ }^{10}$ also developed pain, which was not dependent on the severity of motor deficits. In the study of Cheshire et $a l^{11}$ pain was not associated with functional outcome. Clinical studies and selective lesions of sensory pathways by surgical procedures have shown that central pain is most likely to occur after interruption of spinothalamic tract. ${ }^{17}$ All patients who developed pain had an anterior spinal artery infarct with spinothalamic sensory loss, 
and the three patients who had no spinothalamic sensory loss did not develop pain. However, pain occurred in only 10 of the 25 patients who had a spinothalamic sensory loss. It has been hypothesised that the occurrence of central pain requires a selective lesion of the neospinothalamic tract, sparing spinoreticulothalamic pathways, ${ }^{18}$ for instance in lateral medullary infarcts. ${ }^{19}$ Our findings do not support this hypothesis.

In conclusion, our study showed that the two month outcome mainly depends on the initial severity of the neurological deficits, although a few patients with a severe impairment at onset may have a good outcome, especially when proprioception is spared. Our study does not support the hypothesis that pain occurs more frequently in small infarcts.

\author{
Authors' affiliations \\ C Masson, Department of Neurology, Beaujon Hospital, Clichy, France \\ J P Pruvo, J F Meder, Department of Neuroradiology \\ C Cordonnier, D Leys, Department of Neurology, Lille, France \\ E Touze, J L Mas, Department of Neurology, Paris Ste Anne, France \\ M Giroud, Department of Neurology, Dijon, France \\ $V$ de la Sayette, Department of Neurology, Caen, France
}

\section{APPENDIX}

\section{This study was conducted under the auspice of the French Neurovascular Society}

The French Study Group of Spinal Cord Infarction: Amiens (S Canaple); Angers (I Penisson, F Dubas); Avignon (J Arlaud); Besançon (Th Moulin); Bordeaux (M de Sèze); Caen (V de la Sayette); Calais (O Dereeper); Clichy (C Masson); Dijon (M Giroud); Lille (C Cordonnier, D Leys); Lisieux (F Bouvier); Montbelliard (M Bataillard); Paris Sainte-Anne (E Touzé, JL Mas); Paris Val de Grace (D Bequet); Quimper (P Diraison); Rennes (JF Pinel); Saint-Nazaire (C Bertout).

\section{REFERENCES}

1 Gutowski NJ, Murphy RP, Beale DJ. Unilateral upper cervical posterior spinal artery syndrome following sneezing. I Neurol Neurosurg Psychiatry 1992;55:841-3.

2 Kaneki M, Inove K, Shimizu T, et al. Infarction of the unilateral posterior horn and lateral column of the spinal cord with sparing of posterior columns: demonstration by MRI. J Neurol Neurosurg Psychiatry 1994;57:629-31.

3 Masson C, Berthelot JL, Verstichel $P$, et al. Hémiparésie crurale ataxique par infarctus artériel spinal postérieur. Rev Neurol 1997;153:65-6.

4 Goldsmith $\mathrm{P}$, Rowe $\mathrm{D}$, Jager $\mathrm{R}$, ef al. Focal vertebral artery dissection causing Brown-Sequard's syndrome. J Neurol Neurosurg Psychiatry 1998;64:415-16.

5 de la Sayette V, Leproux F, Letellier P. Cervical cord and dorsal medullary infarction presenting with retro-orbital pain. Neurology 1999;53:632-4.

6 de la Sayette V, Scheaffer S, Coskun O, et al. Cluster headache-like attack as an opening symptom of a unilateral infarction of the cervical cord: persistent anaesthesia and dysaesthesia to cold stimuli. J Neurol Neurosurg Psychiatry 1999;66:397-400.

7 Masson C, Boukriche Y, Berthelot JL, et al. Vertebra, rib and spinal cord infarction probably caused by fibrocartilaginous embolism. Cerebrovasc Dis $2001 ; 12: 142-3$.

8 Masson C, Colombani JM. Unilateral infarct in the posterior artery territory presenting as a "clumsy hand". J Neurol 2002;249:1327-8.

9 Monteiro L, Leite I, Almeida Pinto J, et al. Spontaneous thoracolumbar spinal cord infarction: report of six cases. Acta Neurol Scand 1992;86:563-6.

10 Pelser H, van Gijn J. Spinal infarction. A follow-up study. Stroke 1993;24:896-989.

11 Cheshire WP, Santos CC, Massey EW, et al. Spinal cord infarction: etiology and outcome. Neurology 1996;47:321-30.

12 Salvador de la Barrera S, Barca-Buyo A, Montoto-Marques A, et al. Spinal cord infarction: prognosis and recovery a series of 36 patients. Spinal Cord 2001;39:520-5.

13 Corbin JL. Anatomie et pathologie artérielle de la moelle. Paris: Masson, 1961.

14 Weidauer S, Nichtweiss M, Lanfermann $\mathrm{H}$, et al. Spinal cord infarction: MR imaging and clinical features in 16 cases. Neuroradiology 2002;44:851-7.

15 Mawad ME, Riverta V, Crawford S, et al. Spinal cord ischemia after resection of thoracoabdominal aortic aneurysms: MRI findings in 24 patients. AJNR 1990;11:987-91.

16 Thron AK. Vascular anatomy of the spinal cord. Neuroradiological investigations and clinical syndromes. Springer-Verlag. Berlin, 1988.

17 Davidoff R A. The dorsal columns. Neurology 1989;39:1377-85.

18 Nathan PW, Smith MC, Cook AW. Sensory effects in man of lesions of the posterior columns and of some other afferent pathways. Brain 1986;109:1003-41.

19 MacGowan DJL, Janal MN, Clark WC, et al. Central poststroke pain and Wallenberg's lateral medullary infarction: frequency, character, and determinants in 63 patients. Neurology 1997;49:120-5. 